\title{
Nonalcoholic Fatty Liver Disease and Diabetes: An Epidemiological Perspective
}

\author{
Eun-Jung Rhee
}

Department of Endocrinology and Metabolism, Kangbuk Samsung Hospital, Sungkyunkwan University School of Medicine, Seoul, Korea

Nonalcoholic fatty liver disease (NAFLD) is thought to stem from the body's inability to store excess energy in adipocytes; as such, it is commonly viewed as the hepatic manifestation of metabolic syndrome. The pathogenesis of NAFLD involves ectopic fat accumulation, which also takes place in the liver, muscle and visceral fat. NAFLD is rapidly becoming more widespread in Korea, with an estimated prevalence of $30 \%$ in adults. Type 2 diabetes mellitus (T2DM) and NAFLD share insulin resistance as a common pathophysiological mechanism, and each of these two diseases affects the development of the other. Recent studies have suggested that NAFLD is often present as a comorbidity in T2DM patients. The mutual interrelationship between these conditions is shown by findings suggesting that T2DM can exacerbate NAFLD by promoting progression to nonalcoholic hepatosteatosis or fibrosis, while NAFLD causes the natural course of diabetic complications to worsen in T2DM patients. It remains unknown whether one disease is the cause of the other or vice versa. In this review, I would like to discuss current epidemiological data on the associations between NAFLD and T2DM, and how each disease affects the course of the other.

Keywords: Non-alcoholic fatty liver disease; Diabetes mellitus, type 2; Insulin resistance; Epidemiology

\section{INTRODUCTION}

Nonalcoholic fatty liver disease (NAFLD), defined as more than $5 \%$ of fat infiltration in the liver without alcohol consumption exceeding $30 \mathrm{~g} /$ day in men and $20 \mathrm{~g} /$ day, in women thought to be caused by abdominal obesity and insulin resistance [1]. Histologically, NAFLD is a broadly defined condition, with a spectrum extending from nonalcoholic isolated steatosis or fatty liver to nonalcoholic steatohepatitis (NASH)-related cirrhosis. The global spread of sedentary lifestyle patterns has made NAFLD increasingly common, paralleled by the increase of obesity. The prevalence of NAFLD differs by ethnic group and

Received: 25 August 2019, Revised: 5 September 2019,

Accepted: 9 September 2019

Corresponding author: Eun-Jung Rhee

Department of Endocrinology and Metabolism, Kangbuk Samsung Hospital, Sungkyunkwan University School of Medicine, 29 Saemunan-ro, Jongno-gu, Seoul 03181, Korea

Tel: +82-2-2001-2485, Fax: +82-2-2001-1588, E-mail: hongsiri@hanmail.net diagnostic method, ranging from $15 \%$ to $70 \%$ [2]. NAFLD is driven by ectopic fat accumulation in the liver, and the presence of NAFLD is an indicator of insulin resistance and signals the possibility of ectopic fat accumulation in inappropriate parts of the body, such as visceral fat and the intramuscular, perivascular, and pericardial regions [3]. Therefore, NAFLD is often viewed as the hepatic manifestation of metabolic syndrome (MS); furthermore, it is a major risk factor for type 2 diabetes mellitus (T2DM) and is commonly found as a comorbidity in patients with T2DM [4].

The exact pathophysiological mechanism underlying the reciprocal influence of NAFLD and diabetes has not been conclu-

\section{Copyright $(92019$ Korean Endocrine Society}

This is an Open Access article distributed under the terms of the Creative Commons Attribution Non-Commercial License (http://creativecommons.org/ licenses/by-nc/4.0/) which permits unrestricted non-commercial use, distribution, and reproduction in any medium, provided the original work is properly cited. 
sively established. In this review, I would like to discuss the current epidemiological data regarding the association of NAFLD with T2DM, focusing on data from Asian regions. Some of the studies reported in this review analyzed data from the Kangbuk Samsung Health Study (KSHS), among the largest health screening cohorts worldwide. In addition, this review draws upon a previous article by the author [5].

\section{PREVALENCE OF NAFLD AND ITS RELATIONSHIP WITH METABOLIC RISK FACTORS}

Globally, the prevalence of NAFLD is on the rise due to the rapidly increasing prevalence of obesity and obesity-related comorbidities [6]. NAFLD is the most frequent cause of elevated liver enzymes in countries at different levels of development $[6,7]$. However, variation is present in the estimated prevalence of NAFLD both across populations and as a result of differences in diagnostic test used. The worldwide prevalence of NAFLD is estimated to be as high as 1 billion, and NAFLD is the most widespread cause of chronic liver disease in the United States, affecting 80 to 100 million individuals, of whom NASH is present in almost 25\% [8]. In the Dallas Heart Study, in which hepatic triglyceride content was measured in 2,287 participants in a multiethnic, population-based sample with proton magnetic resonance spectroscopy, significant variation in the prevalence of hepatic steatosis was found according to sex ( $42 \%$ and $24 \%$ in white men and women, respectively) and ethnicity (45\%,33\%, and 24\% in Hispanics, whites, and blacks, respectively) [2].

Early epidemiological studies using data from the KSHS reported information on the prevalence of NAFLD in Asians. The Kangbuk Samsung Health Screening Center performs more than 120,000 health screenings every year, and the total number of participants in this cohort is over a few millions. It is thought to be the largest health screening cohort in the world comprising patients treated at a single center. In a study published in 2006 that analyzed 4,967 participants in the KSHS, when adjusted for age, the prevalence of NAFLD was $16.1 \%$ in the overall study population, $11.2 \%$ in women, and $21.6 \%$ in men [9]. In another cross-sectional study by Jeong et al. [10] that analyzed 140,000 KSHS participants, the prevalence of NAFLD was reported to be $25.2 \%$, with differences by regions; for example, there was a higher prevalence in Gyeonggi province, which is a rural area, than in the Seoul area $(27.7 \%$ vs. $26.9 \%)$.

Many studies have investigated the relationships of NAFLD with metabolic risk factors. In a cross-sectional study, Park et al.
[11] analyzed 120 NAFLD patients and 240 control participants in the KSHS. Of the numerous factors that they investigated, insulin resistance - measured using the homeostasis model assessment of insulin resistance - was the strongest predictor of NAFLD, followed by C-reactive protein levels. In another retrospective study performed in 4,954 KSHS participants, the association between baseline insulin concentrations and NAFLD development after 4 years was assessed [12]. High baseline insulin levels were associated with a 1.2-fold increased risk of NAFLD after 4 years, and the risk increased to 2.5 -fold in participants who had both high insulin levels at baseline and 4 years later. In a study by Yun et al. [13], large baseline waist circumference was shown to increase the risk for NAFLD after 2 years in 37,120 KSHS participants.

Other studies have explored the associations of NAFLD with risk factors beyond the anthropometric parameters discussed above. In 9,162 nondiabetic participants in the KSHS, the relationship of the apolipoprotein (Apo) A1/B ratio with NAFLD was analyzed, and individuals with the lowest quartile of the ApoA1/B ratio showed a 3.5-fold higher risk of developing NAFLD than the highest quartile group [14]. In other studies that analyzed the relationship between serum adipocytokine levels and future NAFLD development, high baseline levels of tumor-necrosis factor-alpha and fatty-acid binding protein-4 increased the risk for NAFLD development after 4 years $[15,16]$.

Regarding lifestyle habits and NAFLD risk, among 120,000 KSHS participants, those who exercised more than five times a week showed a $14 \%$ lower risk for fatty liver development than those who did not exercise at all, and individuals with an increasing amount of exercise during 5 years showed a $13 \%$ higher probability of disappearance of fatty liver than those with a decreasing amount of exercise [17]. Bae et al. [18] conducted a cross-sectional study showing that those who exercised regularly had a lower NAFLD risk than those who did not exercise, regardless of body mass index.

Studies have also investigated the associations of NAFLD with sedentary lifestyle habits and sleep. Kim et al. [19] reported a 59\% higher risk of NAFLD in those who reported sleeping for less than 5 hours per day than in those who slept for over 7 hours per day, through an analysis of 69,493 participants in the KSHS. Furthermore, a recent study of 139,056 participants in the KSHS found that those who engaged in sitting for more than 10 hours a day and those who were inactive showed a higher NAFLD risk than their counterparts who reported sitting less than 5 hours and were active [20]. 


\section{PREVALENCE OF NAFLD IN PATIENTS WITH T2DM}

NAFLD is much more highly prevalent in T2DM patients than in the general population. Lonardo et al. [21] reported in a systematic review that NAFLD was present in $50 \%$ to $75 \%$ of T2DM patients, with variation according to ethnicity. Conversely, diabetes is also more highly prevalent in NAFLD patients than in the general population. Browning et al. [2] used H-NMR spectroscopy to measure hepatic triglyceride content in 2,287 urban residents who participated in the Dallas Heart Study, and found a prevalence of $18 \%$ to $33 \%$ of T2DM or impaired fasting glucose (IFG) in NAFLD patients.

Variation in diagnostic methods may account for discrepancies across studies regarding the prevalence of NAFLD in T2DM patients [5,22]. Classifying NAFLD based on plasma alanine aminotransferase levels yielded a $20.3 \%$ prevalence of NAFLD, whereas using magnetic resonance spectroscopy as a diagnostic tool resulted in a prevalence of $72.8 \%$. Furthermore, the severity of obesity showed a significant relationship with the risk of prevalent NAFLD in T2DM patients [23].

Asians show a comparable prevalence of NAFLD to that of Caucasians. Ultrasonography detected NAFLD in $63.3 \%$ of 929 Korean T2DM patients seen at a university-based diabetes clinic [24]. A study conducted in Japan among 8,352 patients who received health check-ups between 2009 and 2010 suggested that the prevalence of NAFLD may show a relationship with glycemic status, as NAFLD was detected in $25.6 \%, 56.2 \%$, and $68 \%$ of patients with normal fasting glucose, IFG, and T2DM (defined as a fasting blood glucose $\geq 126 \mathrm{mg} / \mathrm{dL}$ ), respectively [25].

\section{NAFLD INCREASES THE RISK FOR T2DM}

Compelling evidence has been found that the development of T2DM is often preceded by NAFLD [26]. Recent findings that NAFLD is an early predictor and determinant of T2DM development have led some researchers to argue that the conventional paradigm according to which NAFLD is the "hepatic manifestation" of MS has become outdated.

An in-depth analysis of the pathophysiology of insulin resistance in the development of NAFLD provides insight into the closely-interwoven relationship of these conditions. Peripheral and hepatic insulin resistance, which are implicated in the development of NAFLD, cause insufficient suppression of hepatic gluconeogenesis, reduced glycogenesis, and higher rates of lipid accumulation [27]. Abundant free fatty acid (FFA) influx from dietary lipids and white adipose tissue into the liver further stimulates gluconeogenesis. On a molecular level, sterol regulatory element binding-protein $1 \mathrm{c}$ and carbohydrate response element binding-protein drive de novo FFA synthesis in response to hyperglycemia and hyperinsulinemia. In insulin-resistant patients, the development of NAFLD and progression to NASH are further induced by factors including increased levels of oxidative stress (caused by the exhaustion of glutamate peroxidase and superoxide dismutase, which function as antioxidants), diacylglycerol-mediated increases in hepatic insulin resistance, and reduced levels of lipid export through very low density lipoprotein [27].

NAFLD has been shown to increase the risk of diabetes by 1.6 to 6.8 times in meta-analyses, with variation according to the other risk factors adjusted for, the diagnostic tools used, and definitions $[28,29]$. In 5,372 Korean participants without diabetes who regularly received health check-ups with a 5 -year period, the relative risk for T2DM was 1.97-fold higher in the entire study population with fatty liver and 2.29-fold higher when frequent drinkers were excluded, compared with those who did not have fatty liver [30]. In another study performed among 11,091 nondiabetic Korean participants in the KSHS who received repeated health screenings with a 5 -year period, those with NAFLD had twice as high of a risk for T2DM as those who did not have NAFLD, and the highest risk was observed in the participants who had the highest insulin levels at baseline [31].

The elevated risk for T2DM could be reduced by resolving NAFLD through weight loss and lifestyle interventions. A study of 13,218 nondiabetic Korean participants in the KSHS divided participants into six groups based on baseline fatty liver status and fatty liver status 5 years later, and assessed the incidence of diabetes in those groups [32]. Using participants with no fatty liver both at baseline and follow-up as the reference group, those who had fatty liver at both timepoints had a 2.78 -fold higher risk for T2DM than the reference group, and a 7.38 times higher risk of T2DM was found in participants in whom fatty liver worsened throughout follow-up. Encouragingly, though, no elevated risk for T2DM was found in participants whose baseline fatty liver had resolved at the 5-year follow-up, implying that their risk of T2DM was attenuated by the resolution of fatty liver. Another study of 4,604 Japanese participants with a 10-year follow-up reported similar results [33]. Furthermore, Bae et al. [34] evaluated the NAFLD status of 7,849 nondiabetic participants who received consecutive annual health check-ups for 5 years. Patients who had NAFLD continuously throughout the 5-year period were at a 1.55 -fold higher risk for T2DM than those who consistently did not have NAFLD. Intriguingly, in- 
termittent NAFLD was not associated with an elevated risk for T2DM, which may indicate that NAFLD sustained over a prolonged time period has a particularly strong impact on T2DM risk.

Certain conditions are known to aggravate the increased risk for T2DM in individuals with NAFLD. A retrospective study of 12,853 Korean KSHS participants without diabetes at baseline found that fatty liver, overweight/obesity, and insulin resistance independently doubled the risk of T2DM [35]. In addition to this remarkable observation, these three conditions seemed to show a synergistic influence on T2DM development, as their coexistence was associated with a 14-fold risk of T2DM. Bae et al. [36] found that concomitant NAFLD and IFG was associated with a nine times higher risk for T2DM after 5 years relative to individuals with neither NAFLD nor IFG at baseline. Furthermore, in patients with MS, NAFLD showed an additive impact on T2DM development, and the presence of increased fibrosis scores or liver enzyme elevation in patients with NAFLD was associated with a higher risk for T2DM [37-39].

As indicated by the findings summarized above, NAFLD can be considered a well-established risk factor for T2DM. Of particular importance is the observation that the resolution of NAFLD reduces T2DM risk. However, T2DM risk is significantly higher when NAFLD is accompanied by hyperglycemia, insulin resistance, or obesity.

\section{T2DM INCREASES THE RISK FOR NASH AND FIBROSIS}

In those with normal liver histology, $10 \%$ to $35 \%$ progress to steatosis. Then, $12 \%$ to $40 \%$ of individuals with hepatic steatosis progress to steatohepatitis, and further progression to cirrhosis occurs in as many of $15 \%$ of NASH patients [40]. The presence of T2DM in subjects with NAFLD may increase their risk for progression to NASH or fibrosis, as well as subsequent development of cirrhosis.

No clear consensus exists regarding factors that contribute to the progression to NASH from NAFLD. In a study of 129 subjects with biopsy-proven NAFLD, but unknown glycemic status at baseline, 78\% had diabetes after 13.7 years of follow-up; moreover, especially prominent insulin resistance was observed in participants with progressive fibrosis (vs. nonprogressive fibrosis), implying that dysglycemia may promote progression to fibrosis [41]. In 1,918 diabetes patients in Hong Kong who received FibroScan examinations, 17.7\% displayed significant liver stiffness [42]. In the Rotterdam study, which included
3,041 participants, individuals with baseline NAFLD and diabetes were significantly more likely than those without either NAFLD or diabetes to have liver stiffness, and NAFLD, diabetes, and age were identified as aggravators of liver stiffness [43]. Another study, which investigated 108 patients who received two liver biopsies at a median 6.6-year interval, the group of patients who showed progression to fibrosis contained a significantly higher percentage of T2DM patients than the group of patients who did not progress to fibrosis [44]. Furthermore, a study that analyzed patients with biopsy-proven NAFLD showed that worsening fibrosis stage (from 0 to 4 ) was associated with a significant increase in the proportion of patients with diabetes, and another study found a converse relationship, in which diabetes was a significant predictor of fibrosis in patients with biopsy-proven NAFLD $[45,46]$.

In light of those findings, in a recent paper, Tilg et al. [27] proposed a clinical algorithm for screening and managing NAFLD, according to which patients with NAFLD should receive screenings for T2DM and MS; furthermore, because T2DM is associated with a significantly elevated risk for NAFLD, patients with T2DM should receive screenings for NAFLD regardless of serum liver enzyme levels, and clinicians should consider performing elastography in these patients to detect liver stiffness.

\section{PRESENCE OF NAFLD AGGRAVATES DIABETIC COMPLICATIONS}

Recent evidence suggests that NAFLD may aggravate the complications of diabetes in T2DM patients. In a study of 2,839 patients with T2DM, NAFLD was associated with a significantly higher prevalence of cardiovascular disease than was found in those without NAFLD, and the risk of macrovascular complications continued to be significantly elevated even after adjusting for several risk factors [47]. In a study of 2,103 patients with NAFLD and T2DM, determined by ultrasonography and their medical history, a 1.87-fold higher risk for chronic kidney disease and a 1.75 -fold higher risk for proliferative retinopathy were found in comparison to patients without NAFLD, implying that NAFLD is associated with a significantly elevated risk for both macrovascular and microvascular complications in patients with T2DM [48].

The results of other studies indicate that concomitant NAFLD and T2DM may be increased with an elevated risk of mortality. Among 337 patients with diabetes who received 10.9 years of follow-up, more liver-related deaths occurred in patients with 


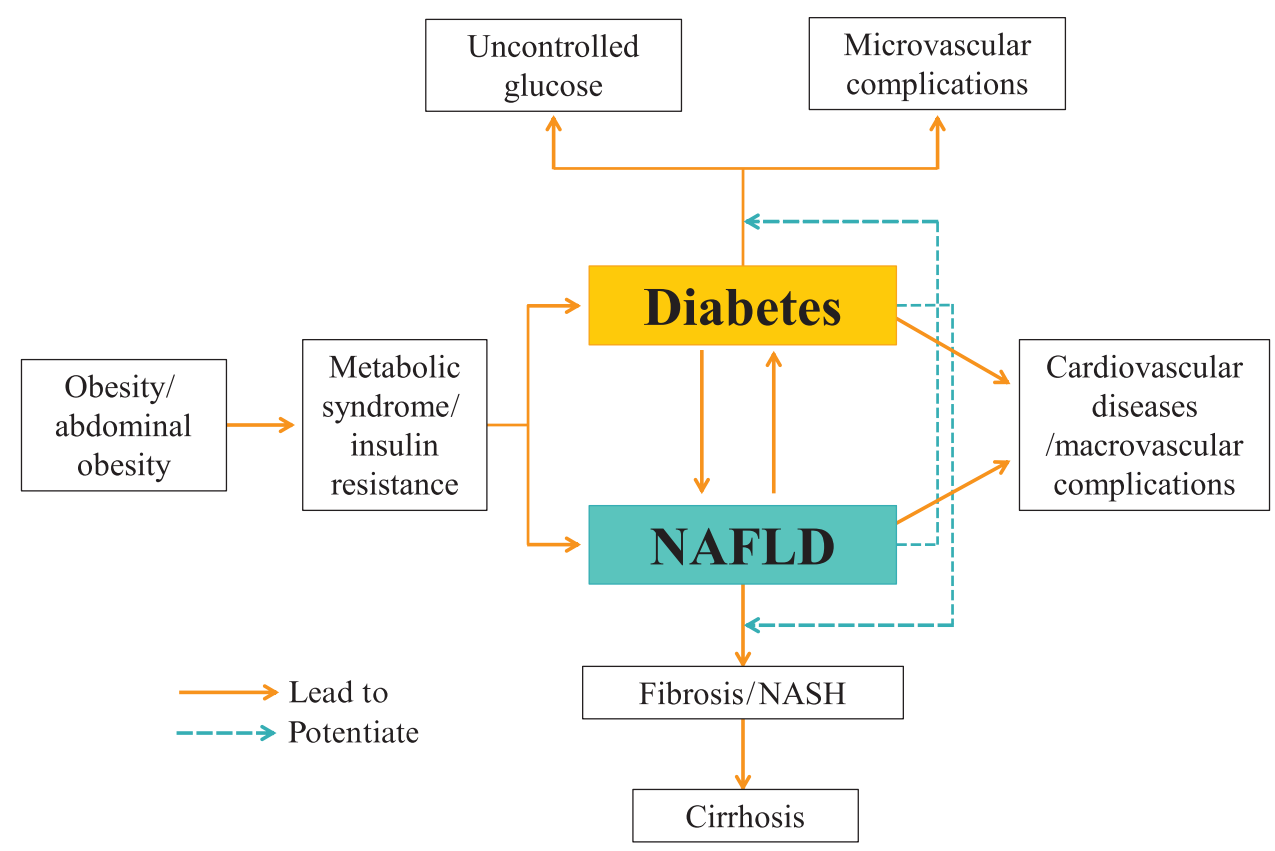

Fig. 1. Relationships between diabetes, nonalcoholic fatty liver disease and their consequences. NASH, nonalcoholic steatohepatitis; NAFLD, nonalcoholic fatty liver disease.

NAFLD than in those without [49]. Furthermore, multivariable analysis identified NAFLD as a significant predictor of mortality in T2DM patients.

An association between concomitant NAFLD and an increased risk of complications of diabetes has also been reported in patients with type 1 diabetes mellitus (T1DM). In a study of 250 patients with T1DM, the prevalence of NAFLD was $44.4 \%$, and both microvascular and macrovascular complications were found to be significantly more common in those with NAFLD than in those without NAFLD [50,51]. These observations imply that NAFLD may exert a deleterious effect on the vasculature, with important consequences for both T1DM and T2DM patients.

\section{CONCLUSIONS}

In this review, I focused on the general epidemiological aspects of NAFLD and its relationship with both T1DM and T2DM. Recent studies have reported strong epidemiological evidence for NAFLD as a risk factor for T2DM, and have also found that T2DM is highly prevalent in patients with NAFLD. Patients with NAFLD who also have T2DM are at an elevated risk for progression to fibrosis and NASH. Conversely, the presence of NAFLD in patients with diabetes aggravates macrovascular and microvascular complications (Fig. 1). The main unsolved prob- lem in research into NAFLD is that there is no single optimal solution that could halt the progression of NAFLD to fibrosis and cirrhosis. Unfortunately, the increasing prevalence of diabetes worldwide may lead to an increasing prevalence of NAFLD. We could encounter serious health problems in the near future caused by unexplained liver-related mortality and morbidity, as well as cardiovascular disease caused by both NAFLD and diabetes. The reciprocal influence of these two diseases on each other could deteriorate the natural course of both conditions. The best solution for preventing this disaster would be treating the fundamental cause of these two diseases. Treatment of obesity, both general and abdominal, via lifestyle modifications and interventions, is likely to be the only fundamental solution for the prevention of these two diseases and their related mortality and morbidity (Fig. 1).

\section{CONFLICTS OF INTEREST}

No potential conflict of interest relevant to this article was reported.

\section{ORCID}

Eun-Jung Rhee https://orcid.org/0000-0002-6108-7758 


\section{REFERENCES}

1. Byrne CD, Targher G. NAFLD: a multisystem disease. J Hepatol 2015;62(1 Suppl):S47-64.

2. Browning JD, Szczepaniak LS, Dobbins R, Nuremberg P, Horton JD, Cohen JC, et al. Prevalence of hepatic steatosis in an urban population in the United States: impact of ethnicity. Hepatology 2004;40:1387-95.

3. Bugianesi E, Gastaldelli A, Vanni E, Gambino R, Cassader $\mathrm{M}$, Baldi $\mathrm{S}$, et al. Insulin resistance in non-diabetic patients with non-alcoholic fatty liver disease: sites and mechanisms. Diabetologia 2005;48:634-42.

4. Marchesini G, Brizi M, Bianchi G, Tomassetti S, Bugianesi E, Lenzi M, et al. Nonalcoholic fatty liver disease: a feature of the metabolic syndrome. Diabetes 2001;50:1844-50.

5. Lee YH, Cho Y, Lee BW, Park CY, Lee DH, Cha BS, et al. Nonalcoholic fatty liver disease in diabetes. Part I: epidemiology and diagnosis. Diabetes Metab J 2019;43:31-45.

6. Chalasani N, Younossi Z, Lavine JE, Diehl AM, Brunt EM, Cusi K, et al. The diagnosis and management of non-alcoholic fatty liver disease: practice guideline by the American Gastroenterological Association, American Association for the Study of Liver Diseases, and American College of Gastroenterology. Gastroenterology 2012;142:1592-609.

7. Das K, Das K, Mukherjee PS, Ghosh A, Ghosh S, Mridha $\mathrm{AR}$, et al. Nonobese population in a developing country has a high prevalence of nonalcoholic fatty liver and significant liver disease. Hepatology 2010;51:1593-602.

8. Loomba R, Sanyal AJ. The global NAFLD epidemic. Nat Rev Gastroenterol Hepatol 2013;10:686-90.

9. Park SH, Jeon WK, Kim SH, Kim HJ, Park DI, Cho YK, et al. Prevalence and risk factors of non-alcoholic fatty liver disease among Korean adults. J Gastroenterol Hepatol 2006; 21(1 Pt 1):138-43.

10. Jeong EH, Jun DW, Cho YK, Choe YG, Ryu S, Lee SM, et al. Regional prevalence of non-alcoholic fatty liver disease in Seoul and Gyeonggi-do, Korea. Clin Mol Hepatol 2013; 19:266-72.

11. Park SH, Kim BI, Yun JW, Kim JW, Park DI, Cho YK, et al. Insulin resistance and $\mathrm{C}$-reactive protein as independent risk factors for non-alcoholic fatty liver disease in non-obese Asian men. J Gastroenterol Hepatol 2004;19:694-8.

12. Rhee EJ, Lee WY, Cho YK, Kim BI, Sung KC. Hyperinsulinemia and the development of nonalcoholic fatty liver disease in nondiabetic adults. Am J Med 2011;124:69-76.

13. Yun KE, Nam GE, Lim J, Park HS, Chang Y, Jung HS, et al.
Waist gain is associated with a higher incidence of nonalcoholic fatty liver disease in Korean adults: a cohort study. PLoS One 2016;11:e0158710.

14. Choe YG, Jin W, Cho YK, Chung WG, Kim HJ, Jeon WK, et al. Apolipoprotein $\mathrm{B} / \mathrm{AI}$ ratio is independently associated with non-alcoholic fatty liver disease in nondiabetic subjects. J Gastroenterol Hepatol 2013;28:678-83.

15. Seo YY, Cho YK, Bae JC, Seo MH, Park SE, Rhee EJ, et al. Tumor necrosis factor- $\alpha$ as a predictor for the development of nonalcoholic fatty liver disease: a 4-year follow-up study. Endocrinol Metab (Seoul) 2013;28:41-5.

16. Jeon WS, Park SE, Rhee EJ, Park CY, Oh KW, Park SW, et al. Association of serum adipocyte-specific fatty acid binding protein with fatty liver index as a predictive indicator of nonalcoholic fatty liver disease. Endocrinol Metab (Seoul) 2013;28:283-7.

17. Sung KC, Ryu S, Lee JY, Kim JY, Wild SH, Byrne CD. Effect of exercise on the development of new fatty liver and the resolution of existing fatty liver. J Hepatol 2016;65:7917.

18. Bae JC, Suh S, Park SE, Rhee EJ, Park CY, Oh KW, et al. Regular exercise is associated with a reduction in the risk of NAFLD and decreased liver enzymes in individuals with NAFLD independent of obesity in Korean adults. PLoS One 2012;7:e46819.

19. Kim CW, Yun KE, Jung HS, Chang Y, Choi ES, Kwon MJ, et al. Sleep duration and quality in relation to non-alcoholic fatty liver disease in middle-aged workers and their spouses. J Hepatol 2013;59:351-7.

20. Ryu S, Chang Y, Jung HS, Yun KE, Kwon MJ, Choi Y, et al. Relationship of sitting time and physical activity with nonalcoholic fatty liver disease. J Hepatol 2015;63:1229-37.

21. Lonardo A, Ballestri S, Marchesini G, Angulo P, Loria P. Nonalcoholic fatty liver disease: a precursor of the metabolic syndrome. Dig Liver Dis 2015;47:181-90.

22. Bril F, Cusi K. Management of nonalcoholic fatty liver disease in patients with type 2 diabetes: a call to action. Diabetes Care 2017;40:419-30.

23. Portillo-Sanchez P, Bril F, Maximos M, Lomonaco R, Biernacki D, Orsak B, et al. High prevalence of nonalcoholic fatty liver disease in patients with type 2 diabetes mellitus and normal plasma aminotransferase levels. J Clin Endocrinol Metab 2015;100:2231-8.

24. Kim BY, Jung CH, Mok JO, Kang SK, Kim CH. Prevalences of diabetic retinopathy and nephropathy are lower in Korean type 2 diabetic patients with non-alcoholic fatty liver 
disease. J Diabetes Investig 2014;5:170-5.

25. Eguchi Y, Hyogo H, Ono M, Mizuta T, Ono N, Fujimoto K, et al. Prevalence and associated metabolic factors of nonalcoholic fatty liver disease in the general population from 2009 to 2010 in Japan: a multicenter large retrospective study. J Gastroenterol 2012;47:586-95.

26. Anstee QM, Targher G, Day CP. Progression of NAFLD to diabetes mellitus, cardiovascular disease or cirrhosis. Nat Rev Gastroenterol Hepatol 2013;10:330-44.

27. Tilg H, Moschen AR, Roden M. NAFLD and diabetes mellitus. Nat Rev Gastroenterol Hepatol 2017;14:32-42.

28. Adams LA, Anstee QM, Tilg H, Targher G. Non-alcoholic fatty liver disease and its relationship with cardiovascular disease and other extrahepatic diseases. Gut 2017;66:113853.

29. Ballestri S, Zona S, Targher G, Romagnoli D, Baldelli E, Nascimbeni F, et al. Nonalcoholic fatty liver disease is associated with an almost twofold increased risk of incident type 2 diabetes and metabolic syndrome: evidence from a systematic review and meta-analysis. J Gastroenterol Hepatol 2016;31:936-44.

30. Kim CH, Park JY, Lee KU, Kim JH, Kim HK. Fatty liver is an independent risk factor for the development of type 2 diabetes in Korean adults. Diabet Med 2008;25:476-81.

31. Sung KC, Kim SH. Interrelationship between fatty liver and insulin resistance in the development of type 2 diabetes. $\mathrm{J}$ Clin Endocrinol Metab 2011;96:1093-7.

32. Sung KC, Wild SH, Byrne CD. Resolution of fatty liver and risk of incident diabetes. J Clin Endocrinol Metab 2013;98: 3637-43.

33. Yamazaki H, Tsuboya T, Tsuji K, Dohke M, Maguchi H. Independent association between improvement of nonalcoholic fatty liver disease and reduced incidence of type 2 diabetes. Diabetes Care 2015;38:1673-9.

34. Bae JC, Han JM, Cho JH, Kwon H, Park SE, Park CY, et al. The persistence of fatty liver has a differential impact on the development of diabetes: the Kangbuk Samsung Health Study. Diabetes Res Clin Pract 2018;135:1-6.

35. Sung KC, Jeong WS, Wild SH, Byrne CD. Combined influence of insulin resistance, overweight/obesity, and fatty liver as risk factors for type 2 diabetes. Diabetes Care 2012;35: 717-22.

36. Bae JC, Rhee EJ, Lee WY, Park SE, Park CY, Oh KW, et al. Combined effect of nonalcoholic fatty liver disease and impaired fasting glucose on the development of type 2 diabetes: a 4-year retrospective longitudinal study. Diabetes Care
2011;34:727-9.

37. Bae JC, Kim SK, Han JM, Kwon S, Lee DY, Kim J, et al. Additive effect of non-alcoholic fatty liver disease on the development of diabetes in individuals with metabolic syndrome. Diabetes Res Clin Pract 2017;129:136-43.

38. Choi JH, Rhee EJ, Bae JC, Park SE, Park CY, Cho YK, et al. Increased risk of type 2 diabetes in subjects with both elevated liver enzymes and ultrasonographically diagnosed nonalcoholic fatty liver disease: a 4-year longitudinal study. Arch Med Res 2013;44:115-20.

39. Chang Y, Jung HS, Yun KE, Cho J, Cho YK, Ryu S. Cohort study of non-alcoholic fatty liver disease, NAFLD fibrosis score, and the risk of incident diabetes in a Korean population. Am J Gastroenterol 2013;108:1861-8.

40. Bhatia LS, Curzen NP, Calder PC, Byrne CD. Non-alcoholic fatty liver disease: a new and important cardiovascular risk factor? Eur Heart J 2012;33:1190-200.

41. Ekstedt M, Franzen LE, Mathiesen UL, Thorelius L, Holmqvist M, Bodemar G, et al. Long-term follow-up of patients with NAFLD and elevated liver enzymes. Hepatology 2006;44:865-73.

42. Kwok R, Choi KC, Wong GL, Zhang Y, Chan HL, Luk AO, et al. Screening diabetic patients for non-alcoholic fatty liver disease with controlled attenuation parameter and liver stiffness measurements: a prospective cohort study. Gut 2016; 65:1359-68.

43. Koehler EM, Plompen EP, Schouten JN, Hansen BE, Darwish Murad S, Taimr P, et al. Presence of diabetes mellitus and steatosis is associated with liver stiffness in a general population: the Rotterdam study. Hepatology 2016;63:13847.

44. McPherson S, Hardy T, Henderson E, Burt AD, Day CP, Anstee QM. Evidence of NAFLD progression from steatosis to fibrosing-steatohepatitis using paired biopsies: implications for prognosis and clinical management. J Hepatol 2015;62:1148-55.

45. Goh GB, Pagadala MR, Dasarathy J, Unalp-Arida A, Sargent R, Hawkins $\mathrm{C}$, et al. Clinical spectrum of non-alcoholic fatty liver disease in diabetic and non-diabetic patients. BBA Clin 2014;3:141-5.

46. Hossain N, Afendy A, Stepanova M, Nader F, Srishord M, Rafiq N, et al. Independent predictors of fibrosis in patients with nonalcoholic fatty liver disease. Clin Gastroenterol Hepatol 2009; 7:1224-9.

47. Targher G, Bertolini L, Rodella S, Tessari R, Zenari L, Lippi $\mathrm{G}$, et al. Nonalcoholic fatty liver disease is independently 
associated with an increased incidence of cardiovascular events in type 2 diabetic patients. Diabetes Care 2007;30: 2119-21.

48. Targher G, Bertolini L, Rodella S, Zoppini G, Lippi G, Day $\mathrm{C}$, et al. Non-alcoholic fatty liver disease is independently associated with an increased prevalence of chronic kidney disease and proliferative/laser-treated retinopathy in type 2 diabetic patients. Diabetologia 2008;51:444-50.

49. Adams LA, Harmsen S, St Sauver JL, Charatcharoenwitthaya $P$, Enders FB, Therneau T, et al. Nonalcoholic fatty liver disease increases risk of death among patients with diabetes: a community-based cohort study. Am J Gastroenterol 2010; 105:1567-73.

50. Targher G, Pichiri I, Zoppini G, Trombetta M, Bonora E. Increased prevalence of cardiovascular disease in type 1 diabetic patients with non-alcoholic fatty liver disease. J Endocrinol Invest 2012;35:535-40.

51. Targher G, Mantovani A, Pichiri I, Mingolla L, Cavalieri V, Mantovani W, et al. Nonalcoholic fatty liver disease is independently associated with an increased incidence of chronic kidney disease in patients with type 1 diabetes. Diabetes Care 2014;37:1729-36. 